\title{
HIERARCHICAL METRICS FOR COMPUTING THE MINIMUM NUMBER OF TEST PATHS IN CONTROL-FLOW GRAPHS
}

\author{
Ronald Salloum \\ California State University, San Bernardino, School of Computer Science and Engineering \\ San Bernardino, CA, 92407, USA
}

\begin{abstract}
The path-based testing of control-flow graphs is an important method for software testing. The minimum number of test paths with respect to different coverage criteria is used as a testability and quality measure of programs. Based on the prime decomposition of control-flow graphs, hierarchical metric formulas have been developed to compute the minimum number of test paths for a given control-flow graph. These recursive formulas have been developed for traditional primes, such as if-then and while statements, and with respect to visit-each-loop, simple path, statement, branch, and basis path (McCabe or structured) coverage. In this paper, we develop formulas for other primes that are available in most programming languages and have become very common in practice. Also, we illustrate an efficient algorithm that utilizes the hierarchical formulas to generate a set with the minimum number of test paths with respect to a given coverage criterion.
\end{abstract}

\section{KEYWORDS}

Software Testing, Path Testing, Control-flow Graph, Prime Decomposition, Hierarchical Metrics

\section{INTRODUCTION}

The control-flow graph (CFG) has been an important model of computer programs in software testing and compiler construction (Ammann et al., 2016; Fenton et al., 2014). A few efficient algorithms and tools for constructing a CFG are available. The path-based testing of CFGs is an important method for software testing and different path coverage criteria have been considered (Ammann et al., 2016; Fenton et al., 2014). The most well-known coverage criteria are as follows (Ammann et al., 2016; Bache et al., 1990; Prather, 1994):

- Visit-each-loop path testing: requires a set of paths of the CFG from the starting node to the end node such that control flows both straight past the loop and around the loop at least once (Bache et al., 1990).

- $\quad$ Simple path testing (no repeated edge): requires a set of paths of the CFG from the starting node to the end node such that every path with no repeated edge is included. Two different definitions of the term "simple path" have appeared in literature: no repeated node (Ammann et al., 2016) and no repeated edge (Bache et al., 1990).

- Branch testing: requires a set of paths of the CFG from the starting node to the end node such that every edge of the graph is covered.

- Statement testing: requires a set of paths of the CFG from the starting node to the end node such that every node of the graph is covered.

- Basis path testing (McCabe or structured testing): requires a set of maximally linearly independent paths from the starting node to the end node of the CFG.

- $\quad$ Prime path testing: require a set of paths from the starting node to the end node of the CFG such that every executable prime path is a subpath of some path in the set. A path is prime if it is a simple path (no repeated node) and is not a proper subpath of another simple path (no repeated node). 
- Edge-pair testing: requires a set of paths from the starting node to the end node of the CFG such that every path of length 2 is covered.

- $\quad$ All path testing: requires the set of all paths from the starting node to the end node of the CFG. This coverage criterion is impractical because it may contain an infinite number of paths.

In this paper, we will consider the first five path coverage criteria. Consider the control flow graph in Figure 1 (Fenton et al., 2014). The set with the minimum number of test paths with respect to each of the five coverage criteria for this control flow graph is as follows:

- Visit-each-loop path testing:

$$
\left\langle E_{2} E_{11}\right\rangle,\left\langle E_{2} E_{10} E_{12} E_{14}\right\rangle,\left\langle E_{2} E_{10} E_{12} E_{13} E_{12} E_{14}\right\rangle,\left\langle E_{1} E_{3} E_{5} E_{6} E_{9}\right\rangle,\left\langle E_{1} E_{3} E_{5} E_{6} E_{7} E_{8} E_{9}\right\rangle,\left\langle E_{1} E_{4} E_{6} E_{9}\right\rangle,\left\langle E_{1} E_{4} E_{6} E_{7} E_{8} E_{9}\right\rangle
$$

- Simple path testing (no repeated edge):

$$
\left\langle E_{2} E_{11}\right\rangle,\left\langle E_{2} E_{10} E_{12} E_{14}\right\rangle,\left\langle E_{1} E_{3} E_{5} E_{6} E_{9}\right\rangle,\left\langle E_{1} E_{3} E_{5} E_{6} E_{7} E_{8} E_{9}\right\rangle,\left\langle E_{1} E_{4} E_{6} E_{9}\right\rangle,\left\langle E_{1} E_{4} E_{6} E_{7} E_{8} E_{9}\right\rangle
$$

- $\quad$ Basis path testing (McCabe or structured testing):

$$
\left\langle E_{2} E_{11}\right\rangle,\left\langle E_{2} E_{10} E_{12} E_{14}\right\rangle,\left\langle E_{2} E_{10} E_{12} E_{13} E_{12} E_{14}\right\rangle,\left\langle E_{1} E_{3} E_{5} E_{6} E_{9}\right\rangle,\left\langle E_{1} E_{3} E_{5} E_{6} E_{7} E_{8} E_{9}\right\rangle,\left\langle E_{1} E_{4} E_{6} E_{9}\right\rangle
$$

- Branch testing:

$$
\left\langle E_{2} E_{11}\right\rangle,\left\langle E_{2} E_{10} E_{12} E_{13} E_{12} E_{14}\right\rangle,\left\langle E_{1} E_{3} E_{5} E_{6} E_{9}\right\rangle,\left\langle E_{1} E_{4} E_{6} E_{7} E_{8} E_{9}\right\rangle
$$

- $\quad$ Statement testing:

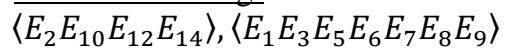

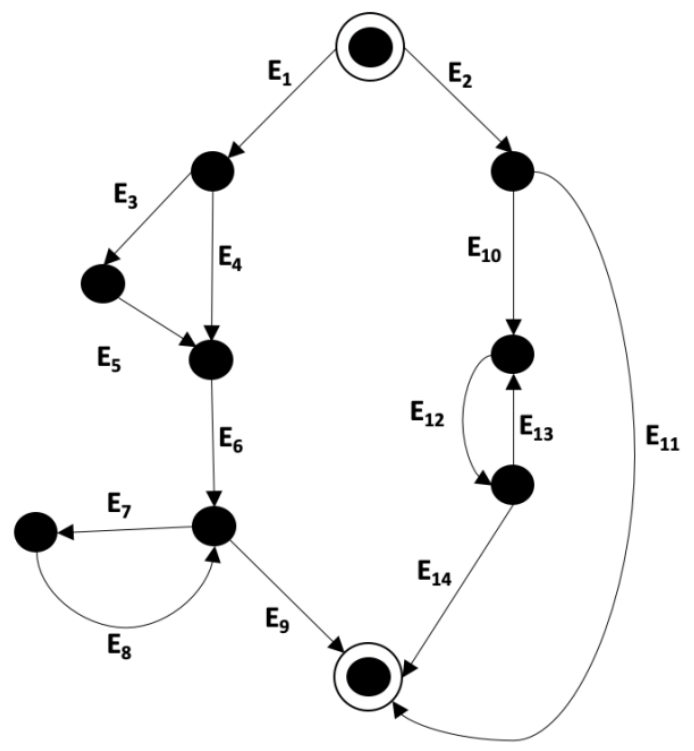

Figure 1. A control flow graph denoted by $G$ with the prime decomposition $G=D_{1}\left(\left(D_{0} ; P ; D_{2}\right), D_{0}\left(D_{3}\right)\right)$

Many different methods, in particular genetic algorithms, have been proposed to automate test data generation for test paths (Bao et al., 2017; Ghiduk, 2014; Khan et al., 2016; Parnami, 2013; Saheed et al., 2014). Some authors have studied the problem of minimizing the number of test paths and selecting the optimal test data (Bures et al., 2019; Prabhu et al., 2011). Hierarchical metrics for CFGs have been used to compute the minimum number of test paths with respect to each of the aforementioned five coverage criteria (Prather, 1994). These formulas for a given CFG are based on the decomposition of the CFG into the traditional primes, namely P: a single or a sequence of arithmetic and/or logical statements (sometimes referred to as a procedure node), $\mathrm{D}_{0}$ : if-then, $\mathrm{D}_{1}$ : if-then-else, $\mathrm{D}_{2}$ : while, and $\mathrm{D}_{3}$ : repeat-until. 
Several other primes, namely while and repeat-until with break, continue, or return statements, have become very common in practice although they violate the traditional structured programming rule (i.e., blocks with one entry and one exit). In this paper, we develop hierarchical metrics for CFGs with these primes for computing the minimum number of test paths with respect to the preceding five coverage criteria. Also, we propose an efficient algorithm for generating a set with the minimum number of test paths with respect to a given coverage criterion.

\section{PRIME DECOMPOSITION}

Programs and their equivalent control flowgraphs are composed of basic statements referred to as primes, such as if-then, if-then-else, while, and repeat-until statements. Four traditional primes are illustrated in Figure 2.

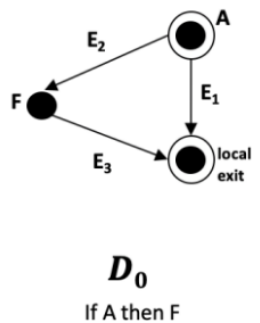

If $A$ then $F$

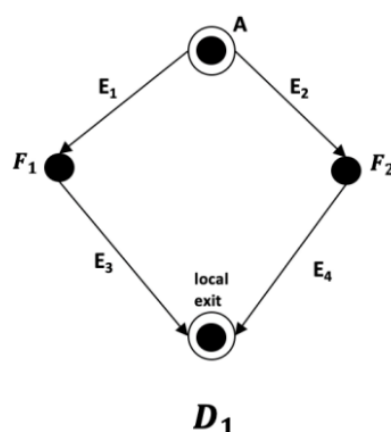

If $A$ then $F_{1}$ else $\mathrm{F}_{2}$

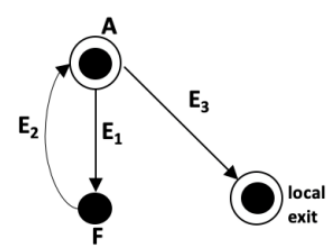

$$
D_{2}
$$

while $A$ do $F$ statements, respectively

Figure 2. Four traditional primes, where $\mathrm{D}_{0}, \mathrm{D}_{1}, \mathrm{D}_{2}$, and $\mathrm{D}_{3}$ represent if-then, if-then-else, while, and repeat-until

There are two basic operations that can be utilized to construct new control-flow graphs from either primes or other control-flow graphs: sequencing and nesting. The sequencing operation is defined as follows. Let $G_{1}$ and $G_{2}$ be two control-flow graphs. Then the sequence of $G_{1}$ and $G_{2}$, denoted by $\left(G_{1} ; G_{2}\right)$, is the control-flow graph formed by merging the exit node of $\mathrm{G}_{1}$ with the starting node of $\mathrm{G}_{2}$. On the other hand, the nesting operation is defined as follows. Suppose $G_{1}$ has a procedure node $F$ (in other words, $F$ does not include a condition). Then the nesting of $G_{2}$ on to $G_{1}$ at $F$, denoted by $G_{1}\left(G_{2}\right.$ on $\left.F\right)$ or more concisely $G_{1}\left(G_{2}\right)$, is the control-flow graph formed from $G_{1}$ by replacing the arc from $F$ with the whole of $G_{2}$. In general, for nesting of $n$ flowgraphs $G_{1}, G_{2}, \ldots G_{n}$ on to $G$, the resulting graph is written as $G\left(G_{1}, G_{2}, \ldots, G_{n}\right)$. Figure 1 shows an example of a control-flow graph that was formed by the sequencing and nesting of primes.

Every program and its equivalent control-flow graph can be represented by its prime decomposition. A prime decomposition of any given graph is formed by performing sequencing and/or nesting of primes. Typically, the prime decomposition is represented by an algebraic expression. For example, for the control flow graph in Figure 1, the prime decomposition is $G=D_{1}\left(\left(D_{0} ; P ; D_{2}\right), D_{0}\left(D_{3}\right)\right)$. Generating the algebraic expression of the prime decomposition of a given program can be easily obtained while constructing the equivalent control-flow graph (Cimitile et al., 1991).

There are many other primes that have become very common in programming practice, but have not been considered in the path testing literature. In this paper, we propose six of these primes: while with return, while with break, while with continue, repeat-until with return, repeat-until with break, and repeat-until with continue. These primes are shown in Figure 3. 

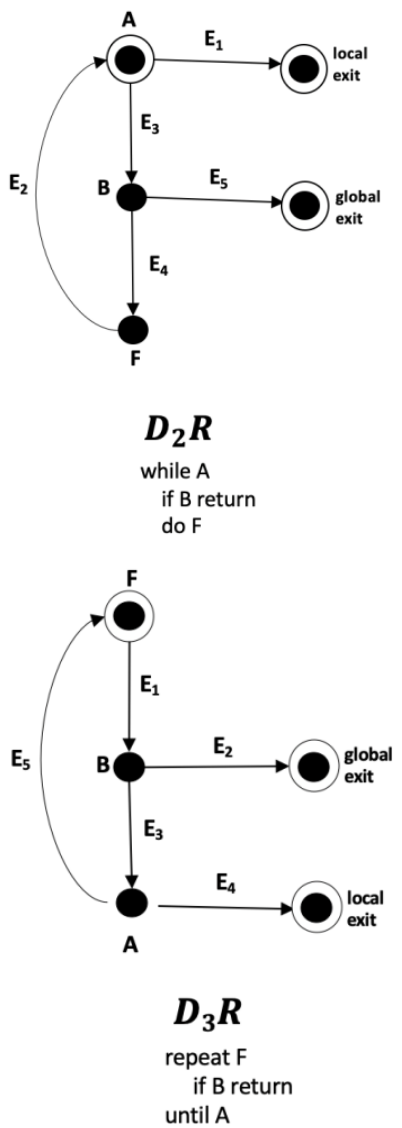
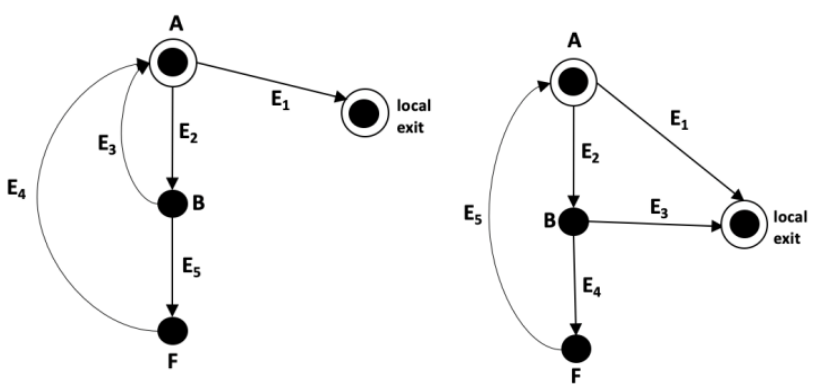

\section{$\mathrm{D}_{2} \mathrm{C}$}

while $\mathrm{A}$ if $B$ continue do $\mathrm{F}$

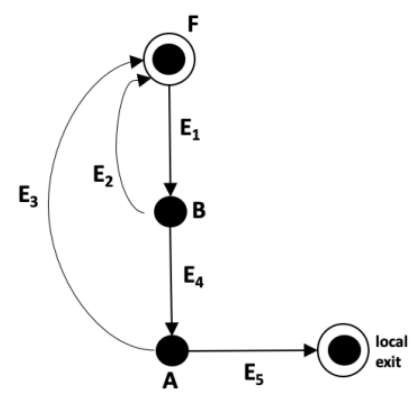

$D_{3} C$

repeat $F$

if $B$ continue

until $A$

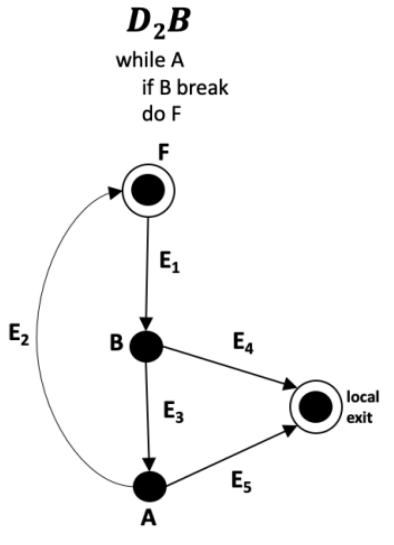

$D_{3} B$

repeat $F$

if $B$ break

until $A$

Figure 3. The six proposed primes. $\mathrm{D}_{2} \mathrm{R}, \mathrm{D}_{2} \mathrm{C}$, and $\mathrm{D}_{2} \mathrm{~B}$ represent while with return, continue, and break statements, respectively. $D_{3} R, D_{3} C$, and $D_{3} B$ represent repeat-until with return, continue, and break statements, respectively

An example of a control-flow graph that includes some of these proposed primes as well as the corresponding prime decomposition is shown in Figure 4.

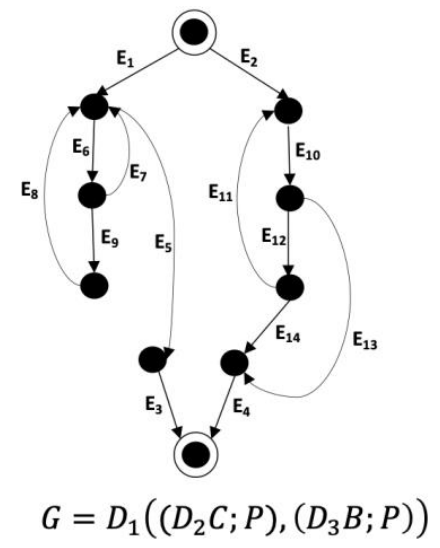

Figure 4. An example of a control-flow graph consisting of some of the proposed primes, as well as the corresponding prime decomposition 


\section{HIEARACHICAL METRICS}

Hierarchical metrics based on the prime decomposition of a given CFG have been used as a measure of software quality and testability. Recursive formulas have been developed for computing the minimum number of test paths with respect to the five coverage criteria (visit-each-loop path testing, simple path testing, branch testing, statement testing, and structured testing). The formulas were developed for control-flow graphs consisting of the traditional primes shown in Figure 2. The minimum number of test paths with respect to the five coverage criteria, denoted by $\mu$, for the traditional primes without and with nesting are shown in Tables 1 and 2, respectively. Table 3 shows the minimum number of paths for sequencing of CFGs.

Table 1. The minimum number of test paths for the traditional primes without nesting

\begin{tabular}{|l|c|c|c|c|c|}
\hline $\begin{array}{l}\text { Test } \\
\text { Strategy }\end{array}$ & $\boldsymbol{\mu}(\boldsymbol{P})$ & $\boldsymbol{\mu}\left(\boldsymbol{D}_{0}\right)$ & $\boldsymbol{\mu}\left(\boldsymbol{D}_{1}\right)$ & $\boldsymbol{\mu}\left(\boldsymbol{D}_{2}\right)$ & $\boldsymbol{\mu}\left(\boldsymbol{D}_{3}\right)$ \\
\hline $\begin{array}{l}\text { Visit-each- } \\
\text { loop path } \\
\text { testing }\end{array}$ & 1 & 2 & 2 & 2 & 2 \\
\hline $\begin{array}{l}\text { Simple } \\
\text { path } \\
\text { testing }\end{array}$ & 1 & 2 & 2 & 2 & 1 \\
\hline $\begin{array}{l}\text { Structured } \\
\text { (basis) } \\
\text { testing }\end{array}$ & 1 & 2 & 2 & 2 & 2 \\
\hline $\begin{array}{l}\text { Branch } \\
\text { testing }\end{array}$ & 1 & 2 & 2 & 1 & 1 \\
\hline $\begin{array}{l}\text { Statement } \\
\text { testing }\end{array}$ & 1 & 1 & 2 & 1 & 1 \\
\hline
\end{tabular}

Table 2. The minimum number of test paths for the traditional primes with nesting

\begin{tabular}{|l|c|c|c|c|}
\hline Test Strategy & $\boldsymbol{\mu}\left(\boldsymbol{D}_{0}(\boldsymbol{F})\right)$ & $\boldsymbol{\mu}\left(\boldsymbol{D}_{1}\left(\boldsymbol{F}_{1}, \boldsymbol{F}_{2}\right)\right)$ & $\boldsymbol{\mu}\left(\boldsymbol{D}_{2}(\boldsymbol{F})\right)$ & $\boldsymbol{\mu}\left(\boldsymbol{D}_{3}(\boldsymbol{F})\right)$ \\
\hline $\begin{array}{l}\text { Visit-each-loop } \\
\text { path testing }\end{array}$ & $\mu(F)+1$ & $\mu\left(F_{1}\right)+\mu\left(F_{2}\right)$ & $\mu(F)+1$ & $\mu(F)+\mu(F)^{2}$ \\
\hline $\begin{array}{l}\text { Simple path } \\
\text { testing }\end{array}$ & $\mu(F)+1$ & $\mu\left(F_{1}\right)+\mu\left(F_{2}\right)$ & $\mu(F)+1$ & $\mu(F)$ \\
\hline $\begin{array}{l}\text { Structured } \\
\text { (basis) testing }\end{array}$ & $\mu(F)+1$ & $\mu\left(F_{1}\right)+\mu\left(F_{2}\right)$ & $\mu(F)+1$ & $\mu(F)+1$ \\
\hline Branch testing & $\mu(F)+1$ & $\mu\left(F_{1}\right)+\mu\left(F_{2}\right)$ & 1 & 1 \\
\hline $\begin{array}{l}\text { Statement } \\
\text { testing }\end{array}$ & $\mu(F)$ & $\mu\left(F_{1}\right)+\mu\left(F_{2}\right)$ & 1 & 1 \\
\hline
\end{tabular}

Table 3. The minimum number of test paths for sequencing of control flowgraphs.

These formulas apply to CFGs containing the traditional primes as well as CFGs containing the six proposed primes

\begin{tabular}{|l|c|}
\hline Test Strategy & $\boldsymbol{\mu}\left(\left(\boldsymbol{G}_{1} ; \ldots ; \boldsymbol{G}_{n}\right)\right)$ \\
\hline $\begin{array}{l}\text { Visit-each-loop path } \\
\text { testing }\end{array}$ & $\prod_{i=1}^{n} \mu\left(G_{i}\right)$ \\
\hline Simple path testing & $\prod_{i=1}^{n} \mu\left(G_{i}\right)$ \\
\hline Structured testing & $\sum_{i=1}^{n} \mu\left(G_{i}\right)-n+1$ \\
\hline Branch testing & $\max \left(\mu\left(G_{1}\right), \ldots, \mu\left(G_{n}\right)\right)$ \\
\hline Statement testing & $\max \left(\mu\left(G_{1}\right), \ldots, \mu\left(G_{n}\right)\right)$ \\
\hline
\end{tabular}

These formulas can be used to determine the minimum number of test paths with respect to any of the five coverage criteria for the prime decomposition of a given graph. For example, consider the prime decomposition of the graph shown in Figure 1. For this prime decomposition, the minimum number of test paths required for the visit-each-loop coverage criteria is given by the following formula:

$$
\begin{aligned}
\mu(G) & =\mu\left(D_{1}\left(\left(D_{0} ; P ; D_{2}\right), D_{0}\left(D_{3}\right)\right)\right) \\
& =\mu\left(\left(D_{0} ; P ; D_{2}\right)\right)+\mu\left(D_{0}\left(D_{3}\right)\right) \\
& =\mu\left(D_{0}\right) \mu(P) \mu\left(D_{2}\right)+\mu\left(D_{3}\right)+1
\end{aligned}
$$




$$
=2 \times 1 \times 2+2+1=7 \text {. }
$$

In this paper, we present the formulas for the minimum number of test paths with respect to the five coverage criteria for the six proposed primes. Tables 4 and 5 give the formulas for the minimum number of test paths for the six proposed primes without and with nesting, respectively. As for sequencing of CFGs, the formulas shown in Table 3 are applicable to the six proposed primes as well.

Table 4. The minimum number of test paths for the six proposed primes without nesting

\begin{tabular}{|l|c|c|c|c|c|c|}
\hline $\begin{array}{l}\text { Test } \\
\text { Strategy }\end{array}$ & $\mu\left(D_{2} R\right)$ & $\mu\left(D_{3} R\right)$ & $\mu\left(D_{2} C\right)$ & $\mu\left(D_{3} C\right)$ & $\mu\left(D_{2} B\right)$ & $\mu\left(D_{3} B\right)$ \\
\hline $\begin{array}{l}\text { Visit-each- } \\
\text { loop path } \\
\text { testing }\end{array}$ & 4 & 4 & 4 & 3 & 4 & 4 \\
\hline $\begin{array}{l}\text { Simple } \\
\text { path } \\
\text { testing }\end{array}$ & 3 & 2 & 3 & 1 & 3 & 2 \\
\hline $\begin{array}{l}\text { Structured } \\
\text { testing }\end{array}$ & 2 & 2 & 3 & 3 & 3 & 3 \\
\hline $\begin{array}{l}\text { Branch } \\
\text { testing }\end{array}$ & 2 & 2 & 1 & 1 & 2 & 2 \\
\hline $\begin{array}{l}\text { Statement } \\
\text { testing }\end{array}$ & 1 & 1 & 1 & 1 & 1 & 1 \\
\hline
\end{tabular}

Table 5. The minimum number of test paths for the six proposed primes with nesting

\begin{tabular}{|l|c|c|c|c|c|c|}
\hline Test Strategy & $\boldsymbol{\mu}\left(\boldsymbol{D}_{2} R(F)\right)$ & $\boldsymbol{\mu}\left(\boldsymbol{D}_{3} \boldsymbol{R}(\boldsymbol{F})\right)$ & $\boldsymbol{\mu}\left(\boldsymbol{D}_{2} \boldsymbol{C}(\boldsymbol{F})\right)$ & $\boldsymbol{\mu}\left(\boldsymbol{D}_{3} \boldsymbol{C}(\boldsymbol{F})\right)$ & $\boldsymbol{\mu}\left(\boldsymbol{D}_{2} \boldsymbol{B}(\boldsymbol{F})\right)$ & $\boldsymbol{\mu}\left(\boldsymbol{D}_{3} \boldsymbol{B}(\boldsymbol{F})\right)$ \\
\hline $\begin{array}{l}\text { Visit-each-loop } \\
\text { path testing }\end{array}$ & $2 \mu(F)+2$ & $\begin{array}{l}2 \mu^{2}(F) \\
+2 \mu(F)\end{array}$ & $2 \mu(F)+2$ & $\begin{array}{l}\mu(F) \\
+2 \mu(F)^{2}\end{array}$ & $2 \mu(F)+2$ & $\begin{array}{l}2 \mu(F)+ \\
2 \mu(F)^{2}\end{array}$ \\
\hline $\begin{array}{l}\text { Simple path } \\
\text { testing }\end{array}$ & $\mu(F)+2$ & $2 \mu(F)$ & $\mu(F)+2$ & $\mu(F)$ & $\mu(F)+2$ & $\mu(F)+1$ \\
\hline Structured testing & $\mu(F)+1$ & $\mu(F)+1$ & $\mu(F)+2$ & $\mu(F)+2$ & $\mu(F)+2$ & $\mu(F)+2$ \\
\hline Branch testing & $\mu(F)+1$ & $\mu(F)+1$ & $\mu(F)$ & $\mu(F)$ & $\mu(F)+1$ & $\mu(F)+1$ \\
\hline Statement testing & $\mu(F)$ & $\mu(F)$ & $\mu(F)$ & $\mu(F)$ & $\mu(F)$ & $\mu(F)$ \\
\hline
\end{tabular}

\section{AN ALGORITHM FOR GENERATING THE TEST PATHS}

In this section, we will present an algorithm for finding a set with the minimum number of test paths with respect to a given coverage criterion, for visit-each-loop path testing, simple path testing, branch testing, and statement testing. Tables 6 and 7 show the actual test paths for the traditional and proposed primes with nesting, respectively. For a given control flowgraph $G$, the notation $S(G)$ represents a set with the minimum number of test paths with respect to a given coverage criterion. For example, in Table 6, $S\left(D_{0}(F)\right)=$ $E_{2} S(F) E_{3} \cup E_{1}$ indicates the set of test paths for the prime $\mathrm{D}_{0}$ with nested $\mathrm{F}$ for the visit-each-loop criterion, where $E_{2} S(F) E_{3}=\left\{E_{2} \alpha E_{3}: \alpha \in S(F)\right\}, E_{1}$ represents a path with a single edge, and $E_{2} S(F) E_{3} \cup E_{1}$ represents the union of these two subsets of paths. Also, $S_{\text {any }}(F) \in S(F)$, which is in some of the entries in Tables 6 and 7, refers to any one path in $S(F)$. 
Table 6. The set of test paths for the four traditional primes with nesting. Please note that if $\mathrm{F}$ is a procedure node, then $\mathrm{S}(\mathrm{F})$ is an empty set

\begin{tabular}{|c|c|c|c|c|}
\hline Test Strategy & $S\left(D_{0}(F)\right)$ & $S\left(D_{1}\left(F_{1}, F_{2}\right)\right)$ & $S\left(D_{2}(F)\right)$ & $S\left(D_{3}(F)\right)$ \\
\hline $\begin{array}{l}\text { Visit-each-loop } \\
\text { path testing }\end{array}$ & $E_{2} S(F) E_{3} \cup E_{1}$ & $E_{1} S\left(F_{1}\right) E_{3} \cup E_{2} S\left(F_{2}\right) E_{4}$ & $E_{1} S(F) E_{2} E_{3} \cup E_{3}$ & $\begin{array}{l}S(F) E_{1} E_{2} \\
\cup S(F) E_{1} E_{3} S(F) E_{1} E_{2}\end{array}$ \\
\hline Simple path testing & $E_{2} S(F) E_{3} \cup E_{1}$ & $E_{1} S\left(F_{1}\right) E_{3} \cup E_{2} S\left(F_{2}\right) E_{4}$ & $E_{1} S(F) E_{2} E_{3} \cup E_{3}$ & $S(F) E_{1} E_{2}$ \\
\hline Branch testing & $E_{2} S(F) E_{3} \cup E_{1}$ & $E_{1} S\left(F_{1}\right) E_{3} \cup E_{2} S\left(F_{2}\right) E_{4}$ & $E_{1} S(F) E_{2} E_{3}$ & $S(F) E_{1} E_{3} S_{\text {any }}(F) E_{1} E_{2}$ \\
\hline Statement testing & $E_{2} S(F) E_{3}$ & $E_{1} S\left(F_{1}\right) E_{3} \cup E_{2} S\left(F_{2}\right) E_{4}$ & $E_{1} S(F) E_{2} E_{3}$ & $S(F) E_{1} E_{2}$ \\
\hline
\end{tabular}

Table 7. The set of test paths for the six proposed primes with nesting. Please note that if $\mathrm{F}$ is a procedure node, then $\mathrm{S}(\mathrm{F})$ is an empty set

\begin{tabular}{|c|c|c|c|c|c|c|}
\hline Test Strategy & $S\left(D_{2} R(F)\right)$ & $S\left(D_{3} R(F)\right)$ & $S\left(D_{2} C(F)\right)$ & $S\left(D_{3} C(F)\right)$ & $S\left(D_{2} B(F)\right)$ & $S\left(D_{3} B(F)\right)$ \\
\hline $\begin{array}{l}\text { Visit-each-loop } \\
\text { path testing }\end{array}$ & $\begin{array}{l}E_{1} \cup E_{3} E_{5} \\
\cup E_{3} E_{4} S(F) E_{2} E_{1} \\
\cup E_{3} E_{4} S(F) E_{2} E_{3} E_{5}\end{array}$ & $\begin{array}{l}S(F) E_{1} E_{2} \cup S(F) E_{1} E_{3} E_{4} \\
\cup S(F) E_{1} E_{3} E_{5} S(F) E_{1} E_{2} \\
\cup S(F) E_{1} E_{3} E_{5} S(F) E_{1} E_{3} E_{4}\end{array}$ & $\begin{array}{c}E_{1} \cup E_{2} E_{3} E_{1} \cup E_{2} E_{5} S(F) E_{4} E_{1} \\
\cup E_{2} E_{3} E_{2} E_{5} S(F) E_{4} E_{1}\end{array}$ & $\begin{array}{l}S(F) E_{1} E_{4} E_{5} \cup S(F) E_{1} E_{2} S(F) E_{1} E_{4} E_{5} \\
\cup S(F) E_{1} E_{4} E_{3} S(F) E_{1} E_{4} E_{5}\end{array}$ & $\begin{array}{l}E_{1} \cup E_{2} E_{3} \\
\cup E_{2} E_{4} S(F) E_{5} E_{1} \\
\cup E_{2} E_{4} S(F) E_{5} E_{2} E_{3}\end{array}$ & $\begin{array}{l}S(F) E_{1} E_{4} \cup S(F) E_{1} E_{3} E_{5} \\
\cup S(F) E_{1} E_{3} E_{2} S(F) E_{1} E_{4} \\
\cup S(F) E_{1} E_{3} E_{2} S(F) E_{1} E_{3} E_{5}\end{array}$ \\
\hline $\begin{array}{l}\text { Simple path } \\
\text { testing }\end{array}$ & $\begin{array}{l}E_{1} \cup E_{3} E_{5} \\
\cup E_{3} E_{4} S(F) E_{2} E_{1}\end{array}$ & $S(F) E_{1} E_{2} \cup S(F) E_{1} E_{3} E_{4}$ & $E_{1} \cup E_{2} E_{3} E_{1} \cup E_{2} E_{5} S(F) E_{4} E_{1}$ & $S(F) E_{1} E_{4} E_{5}$ & $\begin{array}{l}E_{1} \cup E_{2} E_{3} \\
\cup E_{2} E_{4} S(F) E_{5} E_{1}\end{array}$ & $S(F) E_{1} E_{4} \cup S(F) E_{1} E_{3} E_{5}$ \\
\hline Branch testing & $E_{3} E_{4} S(F) E_{2} E_{3} E_{5} \cup E_{1}$ & $\begin{array}{l}S(F) E_{1} E_{3} E_{5} S_{\text {any }}(F) E_{1} E_{2} \\
\cup S_{\text {any }}(F) E_{1} E_{3} E_{4}\end{array}$ & $E_{2} E_{3} E_{2} E_{5} S(F) E_{4} E_{1}$ & $S(F) E_{1} E_{4} E_{3} S_{a n y}(F) E_{1} E_{2} E_{1} E_{4} E_{5}$ & $E_{2} E_{4} S(F) E_{5} E_{1} \cup E_{2} E_{3}$ & $\begin{array}{l}S(F) E_{1} E_{3} E_{2} S_{a n y}(F) E_{1} E_{4} \\
\cup S_{\text {any }}(F) E_{1} E_{3} E_{5}\end{array}$ \\
\hline Statement testing & $E_{3} E_{4} S(F) E_{2} E_{1}$ & $S(F) E_{1} E_{3} E_{4}$ & $E_{2} E_{5} S(F) E_{4} E_{1}$ & $S(F) E_{1} E_{4} E_{5}$ & $E_{2} E_{4} S(F) E_{5} E_{1}$ & $S(F) E_{1} E_{3} E_{2} S_{\text {any }}(F) E_{1} E_{4}$ \\
\hline
\end{tabular}

For a given sequencing of control-flow graphs $\left(G_{1} ; G_{2} ; \ldots G_{n}\right)$, the set of test paths $S\left(\left(G_{1} ; G_{2} ; \ldots G_{n}\right)\right)=$ $S\left(G_{1}\right) S\left(G_{2}\right) \ldots S\left(G_{n}\right)=\left\{\alpha_{1} \alpha_{2} \ldots \alpha_{n}: \alpha_{i}\right.$ is a path in $\left.G_{i}\right\}$ with respect to the visit-each-loop and simple path coverage criteria. In other words, $S\left(\left(G_{1} ; G_{2} ; \ldots G_{n}\right)\right)$ is the set of all possible combination of paths in $G_{1}, G_{2}, \ldots G_{n}$ and its cardinality is equal to $\prod_{i=1}^{n} \mu\left(G_{i}\right)$, as shown in Table 3 .

As for the statement and branch coverage criteria, the cardinality of $S\left(\left(G_{1} ; G_{2} ; \ldots G_{n}\right)\right)$ is equal to $\max \left(\mu\left(G_{1}\right), \ldots, \mu\left(G_{n}\right)\right)$, as shown in Table 3. The set of paths with respect to these two criteria is the set of combination of paths in $G_{1}, G_{2}, \ldots G_{n}$, such that each path in $G_{i}$ is covered at least once. Formally, $S\left(\left(G_{1} ; G_{2} ; \ldots G_{n}\right)\right)=\left\{\alpha_{1} \alpha_{2} \ldots \alpha_{n}: \alpha_{i}\right.$ belongs to $G_{i}$ and every path in $G_{i}$ is covered at least once $\}$.

Given the prime decomposition of a control-flow graph, the algorithm for finding the set with the minimum number of test paths with respect to a given coverage criterion is summarized in the following steps. First, replace each prime with the corresponding set of paths shown in Tables 6 and 7, starting with the outermost primes and proceeding to the innermost primes. Since the edges of the paths in Tables 6 and 7 start at index 1 , then when we replace a prime by its equivalent set of paths, we must adjust the indices so that every prime replacement uses a new set of indices for its edges. For the prime $\mathrm{P}$, it is not necessary to replace it with any edges because it is part of one of the existing edges.

We use the control-flow graph in Figure 4 to illustrate the application of this algorithm. Using the described algorithm, we can generate a set with the minimum number of test paths for the visit-each-loop coverage criterion as follows:

$$
\begin{aligned}
S\left(D_{1}\left(\left(D_{2} C ; P\right),\left(D_{3} B ; P\right)\right)\right) & =E_{1} S\left(\left(D_{2} C ; P\right)\right) E_{3} \cup E_{2} S\left(\left(D_{3} B ; P\right)\right) E_{4} \\
& =E_{1} S\left(D_{2} C\right) S(P) E_{3} \cup E_{2} S\left(D_{3} B\right) S(P) E_{4}
\end{aligned}
$$

We have that $E_{1} S\left(D_{2} C\right) S(P) E_{3}=E_{1}\left(E_{5} \cup E_{6} E_{7} E_{5} \cup E_{6} E_{9} E_{8} E_{5} \cup E_{6} E_{7} E_{6} E_{9} E_{8} E_{5}\right) E_{3}$

$$
=\left\langle E_{1} E_{5} E_{3}\right\rangle,\left\langle E_{1} E_{6} E_{7} E_{5} E_{3}\right\rangle,\left\langle E_{1} E_{6} E_{9} E_{8} E_{5} E_{3}\right\rangle,\left\langle E_{1} E_{6} E_{7} E_{6} E_{9} E_{8} E_{5} E_{3}\right\rangle \text {. }
$$

Also, we have that

$E_{2} S\left(D_{3} B\right) S(P) E_{4}=E_{2}\left(E_{10} E_{13} \cup E_{10} E_{12} E_{14} \cup E_{10} E_{12} E_{11} E_{10} E_{13} \cup E_{10} E_{12} E_{11} E_{10} E_{12} E_{14}\right) E_{4}$ 
$=\left\langle E_{2} E_{10} E_{13} E_{4}\right\rangle,\left\langle E_{2} E_{10} E_{12} E_{14} E_{4}\right\rangle,\left\langle E_{2} E_{10} E_{12} E_{11} E_{10} E_{13} E_{4}\right\rangle,\left\langle E_{2} E_{10} E_{12} E_{11} E_{10} E_{12} E_{14} E_{4}\right\rangle$.

Thus, we conclude that $S\left(D_{1}\left(\left(D_{2} C ; P\right),\left(D_{3} B ; P\right)\right)\right)=$

$\left\langle E_{1} E_{5} E_{3}\right\rangle,\left\langle E_{1} E_{6} E_{7} E_{5} E_{3}\right\rangle,\left\langle E_{1} E_{6} E_{9} E_{8} E_{5} E_{3}\right\rangle,\left\langle E_{1} E_{6} E_{7} E_{6} E_{9} E_{8} E_{5} E_{3}\right\rangle,\left\langle E_{2} E_{10} E_{13} E_{4}\right\rangle,\left\langle E_{2} E_{10} E_{12} E_{14} E_{4}\right\rangle$,

$\left\langle E_{2} E_{10} E_{12} E_{11} E_{10} E_{13} E_{4}\right\rangle,\left\langle E_{2} E_{10} E_{12} E_{11} E_{10} E_{12} E_{14} E_{4}\right\rangle$.

\section{CONCLUSION}

In this paper, we proposed several primes that have become very common in practice but have not been covered in the path testing literature, namely while and repeat-until with break, continue, or return statements. We presented hierarchical formulas for computing the minimum number of test paths for these primes, with respect to visit-each-loop, simple path, statement, branch, and basis path (McCabe or structured) coverage. Also, we presented an efficient algorithm that utilizes these hierarchical formulas to generate a set with the minimum number of test paths with respect to a given coverage criterion (with the exception of the structured coverage criterion). For further research, we suggest investigating the possibility of extending the algorithm to the structured coverage criterion. Also, we suggest extending the results of this paper to other primes and other coverage criteria such as prime path coverage and edge-pair coverage.

\section{REFERENCES}

Ammann, P., \& Offutt, J. (2016). Introduction to software testing. Cambridge University Press.

Bache, R., \& Müllerburg, M. (1990). Measures of testability as a basis for quality assurance. Software Engineering Journal, 5(2), 86-92.

Bao, X., Xiong, Z., Zhang, N., Qian, J., Wu, B., \& Zhang, W. (2017). Path-oriented test cases generation based adaptive genetic algorithm. PloS one, 12(11), e0187471.

Bures, M., \& Ahmed, B. S. (2019). Employment of multiple algorithms for optimal path-based test selection strategy. Information and Software Technology, 114, 21-36.

Cimitile, A., \& De Carlini, U. (1991). Reverse engineering: algorithms for program graph production. Software: Practice and Experience, 21(5), 519-537.

Fenton, N., \& Bieman, J. (2014). Software metrics: a rigorous and practical approach. CRC press.

Ghiduk, A. S. (2014). Automatic generation of basis test paths using variable length genetic algorithm. Information Processing Letters, 114(6), 304-316.

Khan, R., Amjad, M., \& Srivastava, A. K. (2016, February). Optimization of automatic generated test cases for path testing using genetic algorithm. In 2016 Second International Conference on Computational Intelligence \& Communication Technology (CICT) (pp. 32-36). IEEE.

Parnami, S. (2013). Testing target path by automatic generation of test data using genetic algorithm. International Journal of Information and Computation Technology, 3(8), 825-832.

Prabhu, S., Hsiao, M. S., Krishnamoorthy, S., Lingappan, L., Gangaram, V., \& Grundy, J. (2011, November). An efficient 2-phase strategy to achieve high branch coverage. In 2011 Asian Test Symposium (pp. 167-174). IEEE.

[Prather, R. E. (1994, October). Axiomatic foundations for structural software metrics. In Proceedings of 1994 IEEE 2nd International Software Metrics Symposium (pp. 36-44). IEEE.

Saheed, Y. K., \& Babatunde, A. O. (2014). Genetic algorithm technique in program path coverage for improving software testing. Afr J Comp ICT, 7(5), 151-158. 